\title{
Aneurysmal Dermatofibroma of the Earlobe: A Case Report
}

\author{
Daniel Y Xie ${ }^{1}$, Neal Varughese ${ }^{2}$, Kimberly Jared ${ }^{2}$, Richard Bernert ${ }^{3}$, Burrell Wolk ${ }^{2}$ and Neil F Fernandes ${ }^{1,2^{*}}$ \\ ${ }^{1}$ University of Arizona College of Medicine, Phoenix, USA \\ ${ }^{2} S k i n$ and Cancer Center of Arizona, Chandler, USA \\ ${ }^{3}$ Aurora Diagnostics, Scottsdale, USA
}

\begin{abstract}
Aneurysmal dermatofibroma is a rare variant of cutaneous fibrous histiocytoma (dermatofibroma) thought to be secondary to slow extravasation of blood into the lesion. The resulting entities have different clinical and pathological presentations that can lead to diagnostic confusion with other types of skin lesions. We discuss the defining features of aneurysmal dermatofibroma to outline important considerations in the differential to avoid potential misdiagnosis. While dermatofibromas in general tend to follow a benign course, aneurismal dermatofibromas represent a subset with both a higher recurrence rate and a rare risk of metastasis. With these in consideration, proper diagnosis and excision of the lesion should be considered. Although aneurismal dermatofibromas often present on the extremities, few have been reported to appear on other locations of the body. This case report describes an elderly man with an aneurysmal dermatofibroma occurring on the earlobe, a site of occurrence that has only rarely been reported prior.
\end{abstract}

\section{Keywords}

Dermatofibroma, Aneurysmal dermatofibroma, Earlobe

\section{Introduction}

Dermatofibromas are common benign, cutaneous tumors formed by a proliferation of fibroblastic and histiocytic cells [1]. Dermatofibroma can present in patients of all ages, but it is more commonly seen in adults. Per one series, $80 \%$ of biopsy specimens were obtained from patients aged 20 to 49 years, with women more frequently affected than men [2]. In addition, over $70 \%$ of these tumors were located on the extremities [2]. Although dermatofibromas are common in nature, several variants exist with different clinical and histological findings that require careful differentiation to diagnose correctly. Numerous histological variants of dermatofibroma have been described in the literature, including hemosiderotic, epithelioid, cellular, lipidized, atrophic, clear cell, and aneurysmal dermatofibroma [3].

Aneurysmal dermatofibroma is a rare variant that represents less than $2 \%$ of dermatofibromas $[4,5]$. As with dermatofibromas in general, these lesions commonly occur on the extremities and trunks of middle-aged patients, more commonly seen in women [4]. Nonetheless, they can, and have been reported to, occur in other locations [4]. We present a case of an elderly man with aneurysmal dermatofibroma located on the earlobe to highlight the importance of distinguishing this entity from other skin lesions. To the best of our knowledge, our patient represents only the second report of aneurysmal dermatofibroma occurring on the earlobe, the first of which was reported in a female patient in 2013, making it a rare occurrence due to its uncommon location [6].

\section{Case Report}

A 66-year-old man presented with a skin lesion of three years duration on his right earlobe, which had been slowly enlarging and changing. The patient had no relevant medical history and denied family history of melanoma and other skin cancers. Physical examination revealed a $5 \mathrm{~mm}$ reddish, gray, and brown slightly scaly atrophic papule located at the inferior point of attachment of the ear to the cheek (Figure 1).

Shave biopsy was performed, and the specimen was formalin fixed, paraffin embedded, and stained with hematoxylin and eosin. The sample was examined by a board-certified dermatopathologist. Histopathology revealed a cellular dermal proliferation of histiocytic and spindle cells in interlacing bundles within a collagenous stroma with numerous sid-

*Corresponding author: Neil F Fernandes, MD, University of Arizona College of Medicine, Phoenix; Skin and Cancer Center of Arizona, 725 S Dobson Rd Ste 200 Chandler, AZ 85224, USA, Tel: 480-899-7546, Fax: 480-899-7599

Accepted: September 10, 2019

Published online: September 12, 2019

Citation: Xie DY, Varughese N, Jared K, et al. (2019) Aneurysmal Dermatofibroma of the Earlobe: A Case Report. Dermatol Arch 3(1):82-85 
erophages, surrounding cleft-like and pseudovascular spaces. These findings were interpreted as characteristic of aneurysmal dermatofibroma. Subsequent excision of the lesion was performed given the risk, although low, of local recurrence and metastasis. The specimen obtained was similarly formalin fixed, paraffin embedded, and stained with hematoxylin and eosin. The sample was examined independently with identical interpretations by two board-certified dermatopa-

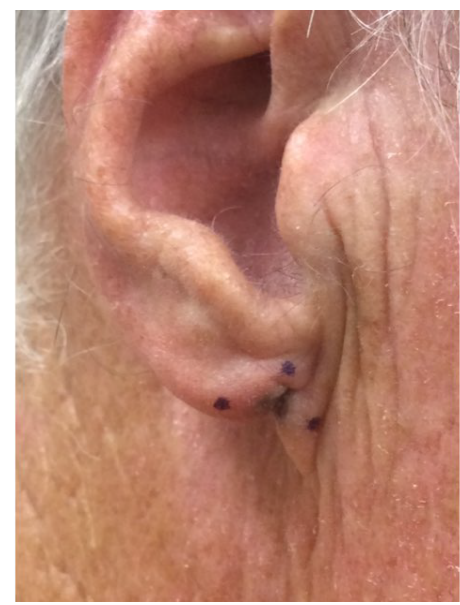

Figure 1: $5 \mathrm{~mm}$ lesion located on right earlobe. Pen marks were placed preoperatively prior to biopsy. thologists. Histopathological analysis of the excision revealed thickening of dermal collagen with jagged border (Figure 2). At higher magnification, trapping of collagen with proliferation of fibroblastic and histiocytic cells was better appreciated (Figure 3A). Presence of pseudovascular spaces was observed on higher magnification (Figure 3B). Higher magnification also revealed deposition of hemosiderin (Figure $3 \mathrm{C}$ ) and the

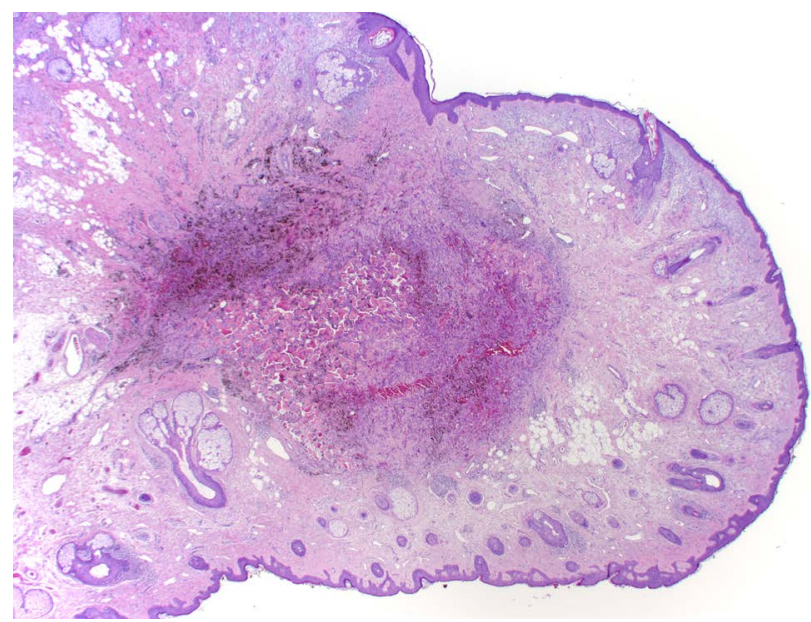

Figure 2: Scanning magnification view showing typical poorly circumscribed nodular architecture with jagged border (hematoxylin and eosin (H\&E), original magnification $\times 20)$.
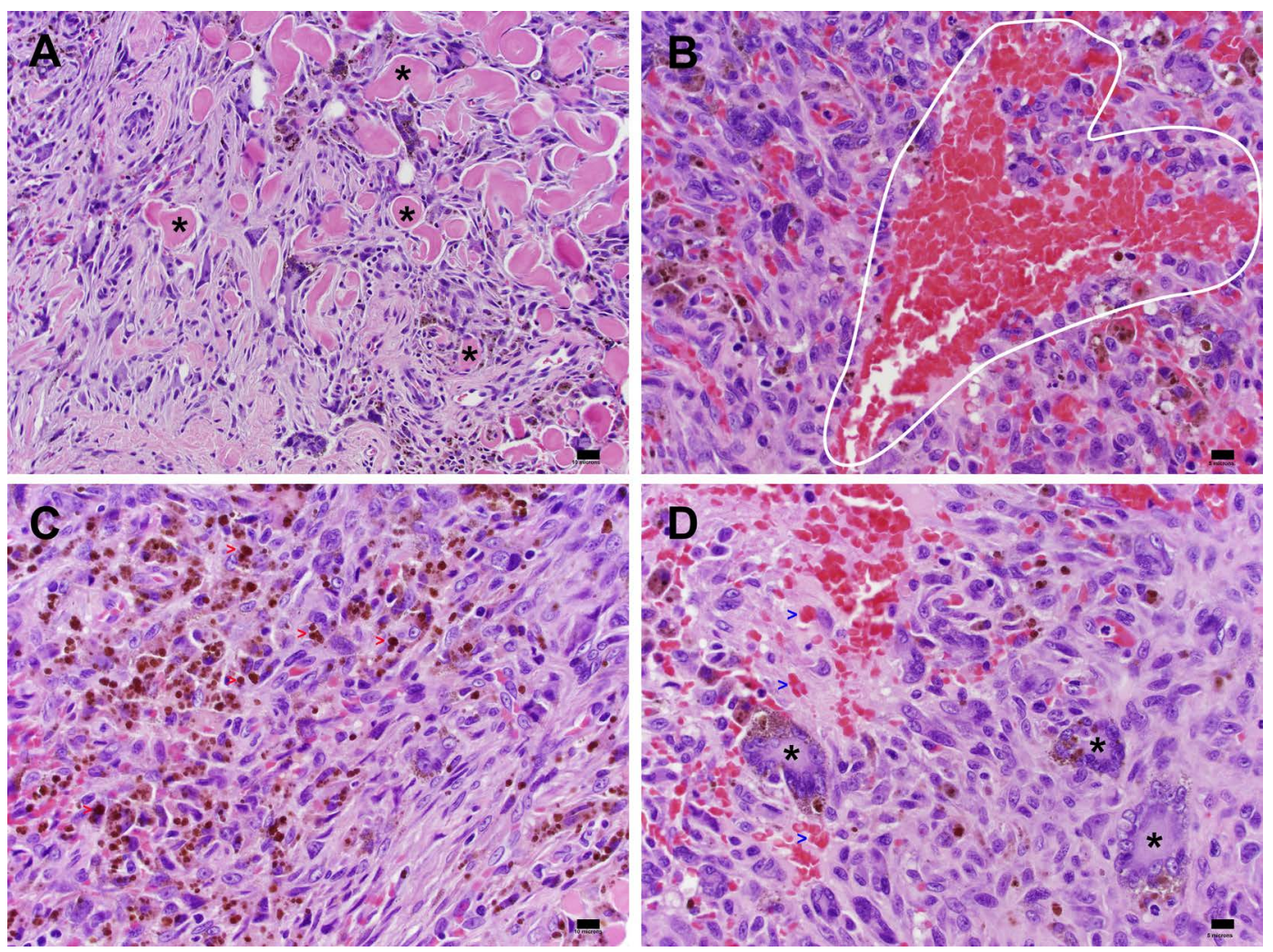

Figure 3: A) Higher power view showing trapping of collagen (asterisks) with proliferation of fibroblastic and histiocytic cells. (H\&E, original magnification $\times 200$ ); B) Enlarged, cavernous blood-filled spaces (white outline) secondary to extravasation of erythrocytes adjacent to mononucleated and multinucleated fibrohistiocytic cells. (H\&E, original magnification $\times 400$ ); C) Deposition of hemosiderin (red arrowheads). (H\&E, original magnification $\times 200$ ); D) Sheets of siderophages, some multinucleated (asterisks), adjacent to extravasated erythrocytes (blue arrowheads). (H\&E, original magnification $\times 400)$. 
presence of multinucleated cells (Figure 3D). These findings matched that of the shave biopsy, confirming the diagnosis of aneurysmal dermatofibroma. No molecular studies were performed for this case.

\section{Discussion}

Clinically, aneurysmal dermatofibromas are characterized by slow-growing, smooth nodular lesions that are cystic in consistency and associated with pain [4,5]. They vary in color, ranging from blue, black, and dark red in early stages to yellow and brown in later stages $[4,5]$. Extravasation of erythrocytes and the presence of hemosiderin play a role in lesion color [4], and variations are likely due to various expressions of red blood cell degradation similar to varying colors of resolving ecchymosis. Additionally, these aneurysmal lesions tend to vary in size between 0.5 and $4 \mathrm{~cm}$, typically larger than more common variants of dermatofibroma (usually measuring 0.5 to $1.5 \mathrm{~cm}$ in size) [5]. In addition, previous history of trauma to the site of the lesion is sometimes reported $[5,7]$.

Histologically, aneurysmal dermatofibroma exhibits typical features of dermatofibroma such as epidermal thickening, dermal proliferation of fibroblastic and histiocytic cells, and peripheral trapping of dermal collagen [8]. Features unique to aneurysmal dermatofibroma include numerous, large pseudovascular spaces lacking endothelial lining. These spaces instead contain numerous fibroblasts and histiocytes, as well as multinucleated giant cells, foam cells, and macrophages [9]. Small capillaries with interstitial hemorrhage are seen leading to deposit and presence of hemosiderin both intracellularly and extracellularly, with high abundance towards the center of the tumor $[8,9]$. Continued extravasation of blood causes tissue spaces to enlarge and form the pseudovascular spaces seen in aneurysmal dermatofibroma [4]. In addition, infiltration of the subcutis has also been observed [8].

Based on clinical and histological findings, features of these lesions can be confused with vascular or malignant tumors; thus, differentiation is important. The differential diagnosis includes Kaposi's Sarcoma, angiomatoid fibrous histiocytoma, hemangiomas, malignant melanoma, and cysts $[4,5]$. In Kaposi's Sarcoma, fibrohistiocytic cells are absent and the primary neoplastic cell is of endothelial origin, which is immunoreactive for CD34 and to a lesser extent, CD31 [4,10-12]. Angiomatoid fibrous histiocytoma, unlike aneurysmal dermatofibroma, tends to have cells that are more epithelioid compared to the spindle cells more commonly found in aneurysmal dermatofibroma [5]. In addition, angiomatoid fibrous histiocytoma occurs exclusively in subcutaneous locations as opposed to aneurysmal dermatofibroma, which is situated predominantly in the dermis (with possible subcutaneous extension) $[8,13,14]$. Hemangiomas lack certain histological features present in aneurysmal dermatofibroma, such as occurrence of fibroblastic and histiocytic cells at the periphery. In addition, hemangiomas lack the presence of both prominent giant cells and foam cells [9]. For malignant melanoma, immunohistochemistry reveals presence of melanin and possible melanophages [15]. Tumor cells in malignant melanoma are often positive for melanocytic markers $\mathrm{HMB}$
45, MITF, MART-1, and S100, allowing for differentiation from aneurysmal dermatofibroma [16-18].

For dermatofibromas in general, a benign course is usually seen with recurrence rates of less than $2 \%$ reported [19]. Thus, treatment is often not required unless symptomatic. Cases of metastasis have been reported for dermatofibromas, though this is a rare occurrence $[8,20,21]$. Although typical dermatofibromas seldom require surgical intervention, excision of the aneurysmal variant should be considered. This is because the aneurysmal variant tends to recur at a higher rate ( $19 \%$ to $20 \%$ ) compared to more common benign fibrous histiocytomas and may also have a low risk of metastasis following incomplete excision $[9,22,23]$.

This case report represents a rare documentation of aneurysmal dermatofibroma appearing on the earlobe. Given the clinical and histological findings, clinicians should be encouraged to consider aneurysmal dermatofibroma as a possibility in the differential diagnosis when lesions of the ear are observed. Recognition of this entity can be important given the minor risk of local recurrence and metastasis.

\section{Financial Disclosures}

The authors report no conflict of interest.

\section{References}

1. Li DF, Iwasaki H, Kikuchi M, et al. (1994) Dermatofibroma: Superficial fibrous proliferation with reactive histiocytes. A multiple immunostaining analysis. Cancer 74: 66-73.

2. Han TY, Chang HS, Lee JH, et al. (2011) A clinical and histopathological study of 122 cases of dermatofibroma (benign fibrous histiocytoma). Ann Dermatol 23: 185-192.

3. Alves JV, Matos DM, Barreiros HF, et al. (2014) Variants of dermatofibroma--a histopathological study. An Bras Dermatol 89: 472-477.

4. Santa Cruz DJ, Kyriakos M (1981) Aneurysmal ("angiomatoid") fibrous histiocytoma of the skin. Cancer 47: 2053-2061.

5. McKenna DB, Kavanagh GM, McLaren KM, et al. (1999) Aneurysmal fibrous histiocytoma: An unusual variant of cutaneous fibrous histiocytoma. J Eur Acad Dermatol Venereol 12: 238-240.

6. López V, Giner F (2013) Lesión nodular en el lóbulo de la oreja de una paciente. Actas Dermosifiliogr 104: 437-438.

7. Kim CJ, Cho JH, Chi JG (1989) Aneurysmal ("angiomatoid") fibrous histiocytoma in a child. J Korean Med Sci 4: 159-162.

8. Luzar B, Calonje E (2010) Cutaneous fibrohistiocytic tumours - an update. Histopathology 56: 148-165.

9. Calonje E, Fletcher CD (1995) Aneurysmal benign fibrous histiocytoma: Clinicopathological analysis of 40 cases of a tumour frequently misdiagnosed as a vascular neoplasm. Histopathology 26: 323-331.

10. Chor PJ, Santa Cruz DJ (1992) Kaposi's sarcoma. A clinicopathologic review and differential diagnosis. J Cutan Pathol 19: 6-20.

11. Russell Jones R, Orchard G, Zelger B, et al. (1995) Immunostaining for CD31 and CD34 in Kaposi sarcoma. J Clin Pathol 48: 10111016.

12. Rosado FG, Itani DM, Coffin CM, et al. (2012) Utility of immunohistochemical staining with FLI1, D2-40, CD31, and CD34 in 
the diagnosis of acquired immunodeficiency syndrome-related and non-acquired immunodeficiency syndrome-related Kaposi sarcoma. Arch Pathol Lab Med 136: 301-304.

13. Costa MJ, Weiss SW (1990) Angiomatoid malignant fibrous histiocytoma. A follow-up study of 108 cases with evaluation of possible histologic predictors of outcome. Am J Surg Pathol 14: 1126-1132.

14. Enzinger FM (1979) Angiomatoid malignant fibrous histiocytoma: A distinct fibrohistiocytic tumor of children and young adults simulating a vascular neoplasm. Cancer 44: 2147-2157.

15. Morariu SH, Suciu M, Vartolomei MD, et al. (2014) Aneurysmal dermatofibroma mimicking both clinical and dermoscopic malignant melanoma and Kaposi's sarcoma. Rom J Morphol Embryol 55: 1221-1224.

16. Fernando SS, Johnson S, Bate J (1994) Immunohistochemical analysis of cutaneous malignant melanoma: Comparison of S-100 protein, HMB-45 monoclonal antibody and $\mathrm{NKI} / \mathrm{C} 3$ monoclonal antibody. Pathology 26: 16-19.

17. Zubovits J, Buzney E, Yu L, et al. (2004) HMB-45, S-100, NK1/C3, and MART-1 in metastatic melanoma. Hum Pathol 35: 217-223.

18. King R, Googe PB, Weilbaecher KN, et al. (2001) Microphthalmia transcription factor expression in cutaneous benign, malignant melanocytic, and nonmelanocytic tumors. Am J Surg Pathol 25: 51-57.

19. Calonje E, Fletcher CDM (1994) Cutaneous fibrohistiocytic tumors: An update. Adv Anat Pathol 1: 2-15.

20. Mentzel T, Wiesner T, Cerroni L, et al. (2013) Malignant dermatofibroma: Clinicopathological, immunohistochemical, and molecular analysis of seven cases. Mod Pathol 26: 256-267.

21. Doyle LA, Fletcher CD (2013) Metastasizing "benign" cutaneous fibrous histiocytoma: A clinicopathologic analysis of 16 cases. Am J Surg Pathol 37: 484-495.

22. Zelger BW, Zelger BG, Steiner H, et al. (1996) Aneurysmal and haemangiopericytoma-like fibrous histiocytoma. J Clin Pathol 49: 313-318.

23. Das A, Das A, Bandyopadhyay D, et al. (2015) Aneurysmal benign fibrous histiocytoma presenting as a giant acrochordon on thigh. Indian Dermatol Online J 6: 436-438. 